\title{
Myanmar Diabetes Care Model: Bridging the Gap Between Urban and Rural Healthcare Delivery
}

\author{
Tint Swe Latt, ${ }^{1}$ Than Than Aye, ${ }^{2}$ Ko Ko, ${ }^{3}$ Ye Myint, ${ }^{4}$ Maung Maung Thant, ${ }^{5}$ Kyar Nyo Soe Myint, ${ }^{5}$ \\ Khin Sanda, ${ }^{5}$ Khaing Lwin, ${ }^{6}$ Htet Htet Khin, ${ }^{5}$ Tin Win Aung, ${ }^{5}$ Kyaw Myint Oo ${ }^{6}$ \\ ${ }^{1}$ Myanmar Diabetes Association \\ ${ }^{2}$ Myanmar Society of Endocrinology and Metabolism \\ ${ }^{3}$ Department of Medicine, University of Medicine 2, Yangon, Myanmar \\ ${ }^{4}$ Department of Medicine, Thingankyun Sanpya Hospital, Yangon, Myanmar \\ ${ }^{5}$ Department of Medicine, North Okkalapa General Hospital, Myanmar \\ ${ }^{6}$ No.2 Military Hospital Yangon, Myanmar
}

\begin{abstract}
There has been significant magnitude of problems of diabetes in Myanmar, according to the estimates of International Diabetes Federation (IDF) and the recent National Survey on the prevalence of diabetes. There has been a wide gap of equity between the urban and rural healthcare delivery for diabetes. Myanmar Diabetes Care Model (MMDCM) aims to deliver equitable diabetes care throughout the country, to stem the tide of rising burden of diabetes and also to facilitate to achieve the targets of the Global Action Plan for the Prevention and Control of NCDs (2013-2020). It is aimed to deliver standard of care for diabetes through the health system strengthening at all level. MMDCM was developed based on the available health system, resources and the country's need. Implementation for the model was also discussed.
\end{abstract}

Key words: diabetes care, NCDs, equity

\section{INTRODUCTION}

Diabetes represents a significant public health burden worldwide by decreasing quality of life and causing death and disability at great economic cost. ${ }^{1}$ Diabetes in all its forms imposes unacceptably high human, social and economic costs on countries at all income levels. ${ }^{2}$

International Diabetes Federation (IDF)'s most recent estimates indicate that $8.3 \%$ of adults -382 million people - have diabetes and the number of people with the disease is set to rise beyond 592 million in less than 25 years. Yet, with 175 million of cases currently undiagnosed, a vast amount of people with diabetes are progressing towards complications unawares. Moreover, with $80 \%$ of the total number affected living in low- and middle-income countries, where the epidemic is gathering pace at alarming rates. ${ }^{2}$

Myanmar is included in the Western Pacific region among the six IDF regions. IDF estimated that the national prevalence of diabetes in 2013 was $5.7 \%$ and total numbers of people with diabetes were $1,988,850$ in

e-ISSN 2308-118X

Printed in the Philippines

Copyright (C) 2015 by the JAFES

Received: October 1, 2015. Accepted: November 26, 2015.

http://dx.doi.org/10.15605/jafes.030.02.16
Myanmar. Numbers of people with diabetes in urban and rural area were estimated as $1,100,380$ and 888,460 respectively. ${ }^{2}$

STEP survey for Yangon Division (2003-3004) reported the prevalence of diabetes as $11.8 \%$ and prevalence of diabetes in urban and rural areas as $13.9 \%$ and $7.3 \%$ respectively. ${ }^{3}$ It was the regional survey conducted in the most populous area of the country, when the Yangon was the capital of the country. There were twice as many people with diabetes as in urban than in rural areas.

National STEP Survey (2009) recorded associated risk factors for diabetes and the prevalence of smoking was $33.6 \%$ in males and $6.1 \%$ in females, the prevalence of hypertension was $31 \%$ in males and $29.3 \%$ in females; the prevalence of overweight was $21.85 \%$ in males and $23.07 \%$ in females; the prevalence of obesity was 4.35 in males and 8.45 in females among the sample population. ${ }^{4}$ Although it was a national survey it included only the survey on the behavioral and physiological risk factors and could not report the prevalence of diabetes which required laboratory measurement of blood glucose. 
National Survey on the prevalence of diabetes and risk factors for non-communicable conducted in 2013-2014 reported the prevalence of diabetes as $10.5 \%$ for the adult population aged between 25 and 65 years. ${ }^{5}$ It also reported the prevalence of risk factors for major non-communicable diseases. The prevalence of hypertension for both sexes was $26.4 \%$. Percentage who currently smokes tobacco was $26.1 \%$ whereas percentage who currently drinks alcohol was $19.8 \%$. The prevalence of overweight (BMI $\left.>25 \mathrm{~kg} / \mathrm{m}^{2}\right)$ and obesity (BMI $>30 \mathrm{~kg} / \mathrm{m}^{2}$ ) were $22.4 \%$ and $5.5 \%$ respectively. ${ }^{5}$ This survey was an extensive survey showing the magnitude of the problem of diabetes and risk factors for major NCDs for the whole country. The survey showed the higher prevalence of diabetes than the estimated prevalence of diabetes by the IDF. Based on this prevalence the total number of diabetes can be more than 2.5 million in Myanmar.

Diabetes mellitus is one of the major non-communicable diseases which also include cardiovascular disease, cancers and chronic respiratory disease. These major noncommunicable diseases share four behavioral risk factors: tobacco use, unhealthy diet and physical inactivity and harmful use of alcohol. Current global mortality from noncommunicable diseases remains unacceptably high and is increasing. Thirty-eight million people each year die from non-communicable diseases. Over 14 million deaths from NCDs occur between the age of 30 and 70 of which $85 \%$ are in the developing countries. ${ }^{6}$

In 2011 September, world leaders adopted the Political Declaration on the non-communicable diseases at the United Nations General Assembly in New York and committed to develop national multi-sectoral action plan for the prevention and control of NCDs and to consider the development of national targets to focus efforts and assess progress made. At the same time, 2011 Political Declaration gave the WHO leadership role along with many time bound assignments. ${ }^{7}$

The World Health Assembly endorsed the WHO Global Action Plan for the Prevention and Control of NCDs 20132020 in May 2013. The Global Action Plan provides Member States, international partners and WHO with a road map and menu of policy options which, when implemented collectively between 2013 and 2020 will contribute to progress on 9 global NCD targets (Appendix 1) to be attained in $2025 .^{8-10}$

A primary healthcare approach is essential to address NCDs effectively and equitably, ${ }^{11,12}$ and the need to strengthen primary care (PC) has been recently highlighted in the political declaration of the United Nations high-level meeting for NCD prevention and control. ${ }^{13}$ There are many cost-effective and high-impact interventions that are feasible to be delivered in PC in lowresource settings by physician as well as non-physician healthcare providers. ${ }^{11}$ These include cardiovascular risk assessment and management to prevent heart attacks and strokes using hypertension and diabetes as entry points, detection, and follow up of diabetes to prevent diabetes complications such as chronic renal disease, smoking cessation counseling to prevent progression of chronic respiratory disease, among others. ${ }^{14-16}$

In Myanmar, WHO Package of Essential NonCommunicable Diseases (PEN) implementation was conducted in two pilot townships in 2012-2013 successfully. Diabetes and hypertension were used as entry point for the screening of high risk persons for cardiovascular diseases at primary care level by Basic Health Staffs who were regularly supervised by township medical officers. Lifestyle modifications like consumption of healthy diet, promotion of physical activity and cessation of tobacco were counseled according to the WHO protocol. Initiation of treatment for diabetes and hypertension to those with high risk of cardiovascular diseases as identified by using WHO/ISH risk score according to the protocol and regular follow up of these patients with counseling on healthy lifestyle and checking the response to treatment with scoring of cardiovascular risk were carried out fortnightly. For successful implementation, community mobilization was undertaken through advocacy of the aim and process of the NCD intervention to the local administrative authorities, community leaders and local NGOs. After three months of implementation, there were improvements in the cardiovascular risk scores with reduction of risk in those patients with high risk of cardiovascular diseases. This pilot project proved the feasibility of implementation of Package of Essential NCD intervention in the low-resource settings in Myanmar. Most importantly there was a building of trust between the community and BHS, which led to the success in undertaking of other public health activities also in the community. ${ }^{17}$

The health system of Myanmar comprises a pluralistic mix of public and private systems both in financing and provision. The network of hospitals and health centers (which extends down to village level) provides preventive and curative services ranging to primary to tertiary care. There are challenges to overcome the limitations of the past (e.g. low investment in rural health services), inadequate funding for expansion of universal coverage and to address health inequities is paramount importance, needing a major reform that will ensure healthcare services to reach the poor and the disadvantaged groups, through the effective functioning of township health system. ${ }^{17}$

Prevention, early detection, diagnosis, and management of NCDs are compromised due to critical health system gaps at PC level. They include deficiencies in equitable health financing, access to medicines and technologies, reliable health information and referral systems, and the health workforce. There is a growing consensus that health 
system strengthening, particularly at PC level is a prerequisite for scaling up prevention and control of NCDs in resource-constrained settings. ${ }^{18}$

The situation of delivery of diabetes care to the public in Myanmar is far from satisfaction. Major challenges in the diabetes care service in Myanmar are public health seeking behaviour issues, presence of traditional medicine, lifestyle and diet issues and issues pertaining to religion and environment. ${ }^{19}$ There is also a wide gap in the equity of delivery of diabetes care between the rural and urban areas. Standard of care for diabetes is available only in cities where majority of physicians and diabetologists work. Essential medication and diagnostic facilities are not available in primary health centers in rural areas where $70 \%$ of the population reside. As the health system is mainly orientated towards prevention and control of communicable diseases and delivery of care for maternal and child health, medical officers and Basic Health Staff needs capacity building for the delivery of standard of diabetes care. Apparently, to improve the delivery of equitable diabetes care to the public, it is utmost important to strengthen the health system with emphasis on the primary healthcare level.

It is imperative to develop Myanmar Diabetes Care Model not only to stem the tide of rising prevalence of diabetes along with its health and socio economic burden but also to achieve the targets set in the Global Action Plan for the prevention and control of NCDs 2013-2020, as a Member State of the World Health Organization as well as the United Nations. Myanmar Diabetes Care Model will be based on the experience of PEN intervention in primary care, using diabetes as the starting point for the screening for high risk for cardiovascular diseases. Moreover, health system strengthening at all level of care will be undertaken and narrowing the gap between the rural and urban delivery of health services with particular focus on the improvement in the diabetes care and integrated approach for the major NCD.

\section{Literature review}

Diabetes can be found in every country. Without effective prevention and management programmes, the burden will continue to increase worldwide. ${ }^{20}$ Diabetes is a complex, chronic illness requiring continuous medical care with multifactoral risk-reduction strategies beyond glycemic control. ${ }^{21}$

American Diabetes Association (ADA) has recommended "standards of medical care in diabetes." ADA position statements on "Standards of Medical Care in Diabetes" provides key clinical practice recommendations which are viewed as important resources for healthcare professionals who care for people with diabetes. ${ }^{21}$

Though quality diabetes care is essential to prevent longterm complications, care often fails below recommended standards regardless of healthcare setting or patient population, emphasizing the necessary for system change. ${ }^{22}$

The chronic care model (CCM) is a multifaceted framework for enhancing healthcare delivery. The model is based on a paradigm shift from the current model of dealing with acute care issues to system that is prevention based. ${ }^{23}$ The premise of the model is that quality diabetes care is not delivered in isolation and can be enhanced by community resources, self-management support, delivery system redesign, decision support, clinical information systems, and organizational support working in tandem to enhance patient -provider interactions. ${ }^{24-26}$ The CCM has been shown to be an effective framework for improving the quality of diabetes care. ${ }^{27}$ Currently, few efforts exist to improve quality of care in diabetes despite studies that demonstrate their proven effectiveness. ${ }^{28}$

A group of external and $\mathrm{WHO}$ experts have developed a guideline for diagnosis and management of type 2 diabetes in primary healthcare in low-resource settings. The primary goal of the guideline is to improve the qualities of care and the outcome for people with type 2 diabetes in low-resource settings. It recommends a set of basic intervention to integrate management of diabetes into primary healthcare (Table 1$){ }^{29}$

Effective approaches to reduce the noncommunicable disease burden in low- and middle-income countries (LMIC) include a mixture of population-wide and individual interventions. Such cost-effective interventions are already available and include methods for early detection of NCDs and their diagnosis using inexpensive technologies, non-pharmacological and pharmacological approaches for modification of NCD risk factors and affordable medications for prevention and treatment of heart attack, strokes, diabetes, cancer and asthma. The WHO Package of Essential Non-communicable Disease Interventions (WHO PEN) for primary care in lowresource settings is an innovative and action-oriented response to the above challenges. It is a prioritized set of cost-effective interventions that can be delivered to an acceptable quality of care, even in resource-poor settings. ${ }^{14}$

In Myanmar, WHO Package of Essential noncommunicable Diseases (PEN) implementation was conducted in two pilot townships in 2012-2013 successfully. Diabetes and hypertension were used as entry point for the screening of high risk persons for cardiovascular diseases at primary care level by Basic Health Staffs who were regularly supervised by township medical officers. Lifestyle modifications like consumption of healthy diet, promotion of physical activity and cessation of tobacco were counseled according to the WHO protocol. Initiation of treatment for diabetes and hypertension to those with high risk of cardiovascular diseases as identified by using WHO/ISH 
ONLINE FIRST | November 30, 2015 | http://dx.doi.org/10.15605/jafes.030.02.16

\begin{tabular}{|c|c|c|c|}
\hline No. & Recommendation & $\begin{array}{l}\text { Quality of } \\
\text { evidence }\end{array}$ & $\begin{array}{c}\text { Strength of } \\
\text { recommendation }\end{array}$ \\
\hline 1 & $\begin{array}{l}\text { Point of care devices can be used in diagnosing diabetes if laboratory services are not } \\
\text { available. }\end{array}$ & not graded & strong \\
\hline 2 & Advise overweight patients to reduce weight by reducing their food intake. & very low & conditional \\
\hline 3 & $\begin{array}{l}\text { Advise all patients to give preference to low glycaemic-index foods (beans, lentils, oats and } \\
\text { unsweetened fruit) as the source of carbohydrates in their diet. }\end{array}$ & moderate & conditional \\
\hline 4 & $\begin{array}{l}\text { Advise all patients to practice regular daily physical activity appropriate for their physical } \\
\text { capabilities (e.g. walking). }\end{array}$ & very low & conditional \\
\hline 5 & $\begin{array}{l}\text { Metformin can be used as a first-line oral hypoglycaemic agent in patients with type } 2 \\
\text { diabetes who are not controlled by diet only and who do not have renal insufficiency, liver } \\
\text { disease or hypoxia. }\end{array}$ & very low & strong \\
\hline 6 & $\begin{array}{l}\text { Give sulfonylurea to patients who have contraindications to metformin or in whom metformin } \\
\text { does not improve glycaemic control. }\end{array}$ & very low & strong \\
\hline 7 & Give a statin to all patients with type 2 diabetes aged $\geq 40$ years. & moderate & conditional \\
\hline 8 & The target value for diastolic blood pressure in diabetic patients is $\leq 80 \mathrm{~mm} \mathrm{Hg}$. & moderate & strong \\
\hline 9 & The target value for systolic blood pressure in diabetic patients is $<130 \mathrm{~mm} \mathrm{Hg}$ & low & weak \\
\hline 10 & $\begin{array}{l}\text { Low-dose thiazides ( } 12.5 \mathrm{mg} \text { hydrochlorothiazide or equivalent) or ACE inhibitors are } \\
\text { recommended as first-line treatment of hypertension in diabetic patients. They can be } \\
\text { combined. }\end{array}$ & $\begin{array}{l}\text { very low for } \\
\text { thiazides, low for } \\
\text { ACE inhibitors }\end{array}$ & strong \\
\hline 11 & $\begin{array}{l}\text { Beta blockers are not recommended for initial management of hypertension in diabetic } \\
\text { patients, but can be used if thiazides or ACE inhibitors are unavailable or contraindicated. }\end{array}$ & very low & strong \\
\hline 12 & $\begin{array}{l}\text { Give patients health education of patients on foot hygiene; nail cutting, treatment of calluses, } \\
\text { appropriate footwear. }\end{array}$ & low & strong \\
\hline 13 & $\begin{array}{l}\text { Educate healthcare workers on assessment of feet at risk of ulcers using simple methods } \\
\text { (inspection, pin-prick sensation) }\end{array}$ & low & strong \\
\hline 14 & $\begin{array}{l}\text { Persons with type } 2 \text { diabetes should be screened for diabetic retinopathy by an } \\
\text { ophthalmologist when diabetes is diagnosed and every two years thereafter, or as } \\
\text { recommended by the ophthalmologist. }\end{array}$ & low & conditional \\
\hline 15 & $\begin{array}{l}\text { Unconscious diabetic patients on hypoglycaemic agents and/or blood glucose } \leq 2.8 \text { should be } \\
\text { given hypertonic glucose intravenously. Food should be provided as soon as the patient can } \\
\text { ingest food safely. }\end{array}$ & strong & strong \\
\hline 16 & $\begin{array}{l}\text { Unconscious diabetic patients on hypoglycaemic agents and/or blood glucose } \leq 2.8 \mathrm{mmol} / \mathrm{L} \\
\text { administer intravenously } 20 \text { to } 50 \mathrm{ml} \text { of } 50 \% \text { glucose (dextrose) over } 1 \text { to } 3 \text { minutes. If not } \\
\text { available, substitute with any hypertonic glucose solution. Food should be provided as soon } \\
\text { asthe patient can ingest food safely. }\end{array}$ & very low & strong \\
\hline 17 & $\begin{array}{l}\text { If blood glucose } \geq 18 \mathrm{mmol} \text { (refer to hospital with i.v. drip } 0.9 \% \mathrm{NaCl} 1 \text { litre in } 2 \text { hours, continue } \\
\text { at } 1 \text { litre every } 4 \text { hours until hospital. }\end{array}$ & very low & strong \\
\hline
\end{tabular}

risk score according to the protocol and regular follow up of these patients with counseling on healthy lifestyle and checking the response to treatment with scoring of cardiovascular risk were carried out fortnightly. For successful implementation, community mobilization was undertaken through advocacy of the aim and process of the NCD intervention to the local administrative authorities, community leaders and local NGOs. After three months of implementation, there were improvements in the cardiovascular risk scores with reduction of risk in those patients with high risk of cardiovascular diseases. This pilot project proved the feasibility of implementation of Package of Essential NCD intervention in the low-resource settings in Myanmar. Most importantly there was a building of trust between the community and BHS, which led to the success in undertaking of other public health activities also in the community. ${ }^{30}$

\section{Myanmar Diabetes Care Model (MMDCM)}

The Diabetes Model of Care provides a framework for comprehensive, accessible and efficient provision of coordinated diabetes prevention and management services for both rural and urban areas throughout the country. It is based not only on the country specific situation especially health system, diabetes, NCDs epidemiology, and the need of the country but also on the literature review in particular on the best practices in the diabetes care and experience with PEN implementation in primary care in pilot townships. It is aimed to deliver comprehensive and equitable healthcare service to those people with prediabetes or diabetes. The unique features of the care model are: empowering the basic health staffs in the diabetes care, community mobilization and participation in the care plan, and application of concepts of Universal Health Coverage (UHC) and WHO's list of essential drugs and integration of diabetes care to the prevention and control of other major non-communicable diseases. It is an effort to fulfill the Myanmar's obligation to the targets and indicators for diabetes as endorsed in the Global Action Plan for the prevention and control of non-communicable diseases (2013-2020). It is expected that the successful implementation of the MMDCM will also assist in achievement of other targets through the adoption of healthy life style throughout the whole country.

\section{Vision}

A nation free of the avoidable burden of diabetes mellitus.

\section{Mission}

Effective and equitable care for people with diabetes both rural and urban areas throughout the country. 


\section{Goal}

To reduce the preventable and avoidable burden of diabetes by delivering the comprehensive healthcare to the people with diabetes through the community mobilization and empowering of basic health staff in the diabetes care throughout the country equitably.

\section{Objectives}

1. Prevention and delay the onset of diabetes in the community by facilitating the adoption of healthy life style;

2. Early detection of diabetes patients by offering screening for asymptomatic patients;

3. Delivery of standard of care for diabetes to those patients equitably throughout the country to prevent and slow the progression of diabetes complications;

4. Establishing the proper referral system for diabetes patients with complications.

\section{Structure of the Diabetes Care Model}

Myanmar healthcare system evolves with changing political and administrative system and relative roles played by the key providers are also changing although the Ministry of Health remains the major provider of comprehensive healthcare. It has a pluralistic mix of public and private system both in the financing and provision. Healthcare is organized and provided by public and private providers. ${ }^{12}$

Structure of the Diabetes Care Model is planned to be aligned with the structure of Myanmar healthcare system which comprises primary, secondary and tertiary care level (Figure 1).
Primary healthcare level consists of townships and rural health centers. There are more than 300 townships throughout the country. Each township has 5-6 rural health centers. Township health center is taken care by township medical officer who is responsible for administration works, clinical care of both outpatients and inpatients, and supervision of public health activities. There are 4-6 medical doctors working in township hospital under the supervision of township medical officer. The main responsibility of township medical center is to offer the general clinical care service and the public health activities such as surveillance and prevention of infectious diseases, maternal and child healthcare, immunization of community according to the extended program of immunization of the country. There is no specialist medical care service available at the township medical health center. Function of the rural health centers are carried out by Basic Health Staff like Health Assistants, Midwives and Public Health Supervisors.

Secondary healthcare level is provided at the district level where specialists medical care services are available. Each district hospital has specialists such as physicians, obstetricians and gynecologists, pediatricians and general surgeons. There are also private clinics and private hospitals in some of the districts.

Tertiary healthcare level is provided at the States and Regions and major cities like Yangon, Mandalay and Naypyidaw. University teaching hospitals are situated mainly in Yangon, Mandalay, Magway and Taunggyi where ultra-specialists care like cardiology, endocrinology, oncology, nephrology etc. are available. Private hospitals with specialist medical care are also available at tertiary healthcare level.

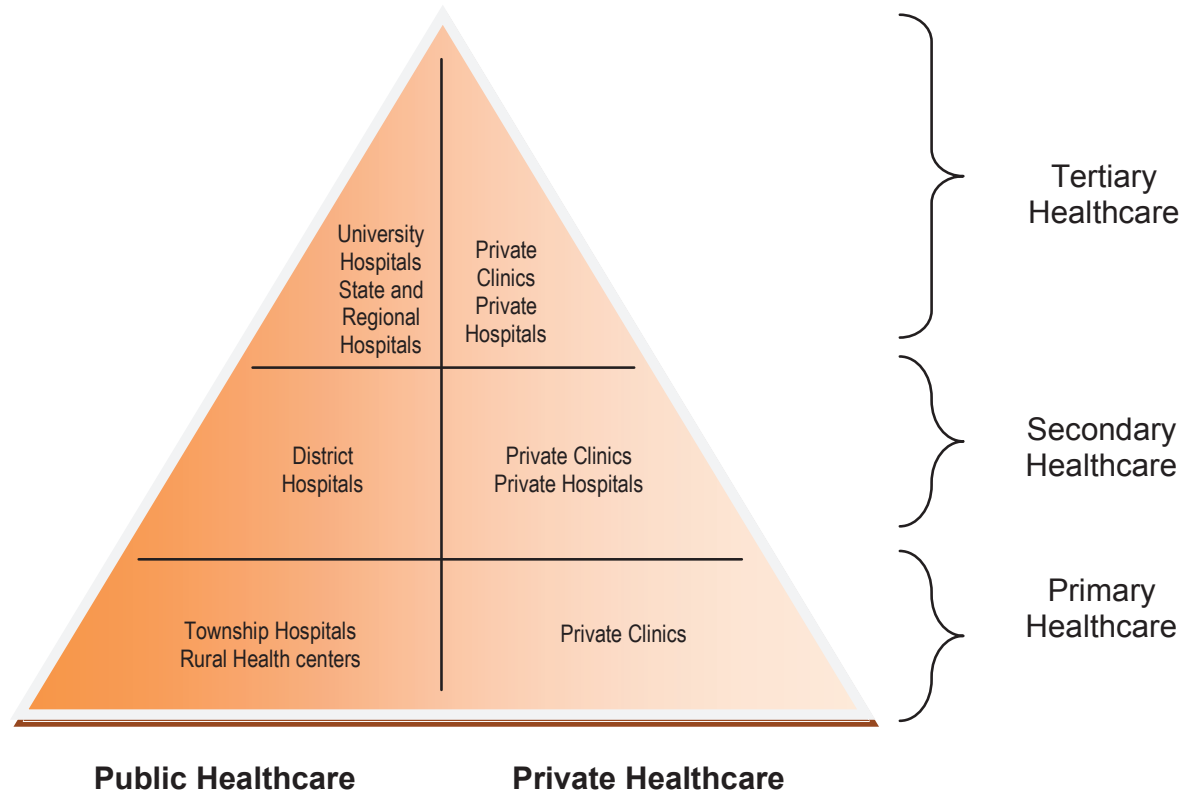

Figure 1. Structure of Myanmar Healthcare system. 


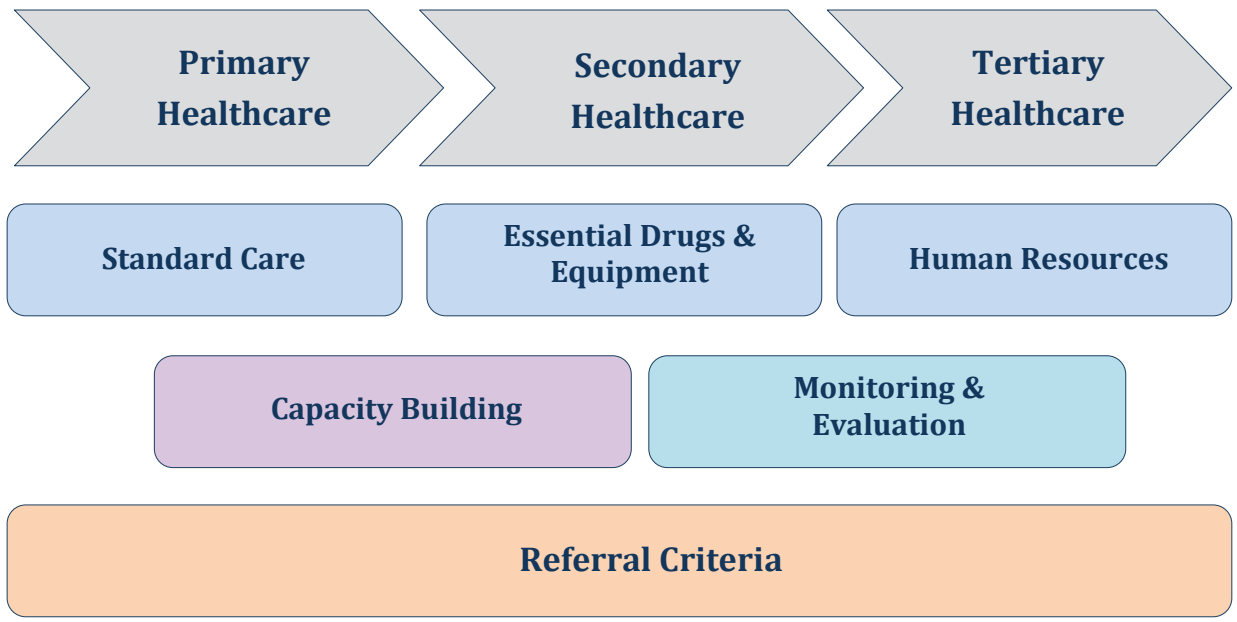

Figure 2. Myanmar Diabetes Care Model.

Diabetes care services will be made accessible from rural health center to tertiary level. Each level will have to perform specified functions for delivery of care in accordance with the guidelines for delivery of standard of diabetes care at different healthcare level. Each level of care will be supported by specified lists of essential drugs and equipment, and required human resources. Private clinics and private hospitals will also be included in the delivery of standard of diabetes care according to the level of healthcare. Basic health staff and medical doctors from township medical hospital, station medical hospital and private clinics will be strengthened their capacities to deliver the standard of care to diabetes patients. Guidelines for proper referral for appropriate care for the diabetes patients will also be included in the capacity building program. Each healthcare center including private clinic will be provided with a set of minimal data for monitoring and evaluation (Figure 2).

At the primary healthcare level, in rural health centers, work done by basic health staff will be supervised by Health Assistants who will in turn be under the supervision of designated medical officer from the township medical center. There are also private clinics which mainly deliver ambulatory care to the community. TMO will overall supervise the activities and monitor the progress of all healthcare centers including private clinics in the township.

Physician of the district hospital will supervise the activities of diabetes care service in the hospital and District Medical Officer will supervise the activities of the delivery of diabetes care in the private clinics in the district.

At the tertiary healthcare level, diabetologists or the senior consultant physicians of the hospital will supervise the activities of diabetes care of the hospitals and public health officers of the States and Region health Office will supervise the activities diabetes care of the private sectors.

\section{Function of diabetes care centers}

Each healthcare center delivering standard of diabetes care will perform the specified functions according to the level of healthcare (Table 2). Management of diabetes and cardiovascular risk factors will be performed according to the set protocol for the primary care (Appendix 2). ${ }^{18}$ At each level of care, health education and counseling on healthy behavior (healthy diet, physical activity, cessation of consumption of tobacco and avoidance of harmful use of alcohol) will be given to all persons attending the health center according to the set protocol (Appendix 3). ${ }^{18}$

Each level of care will be provided with lists of essential drugs and equipment (Table 3) and required health personnels (Table 4).

\section{Implementation}

\section{Funding}

Implementation of the diabetes care model will be in different phases according to the availability of the funding which will be mainly from the government healthcare budget. Resource mobilization will be undertaken from local and international philanthropic organizations and international civic organizations and developmental partners. Since the change in political system to democratic government in 2011 the health expenditure has been increased to $2.4 \%$ of GDP, which is still the lowest among the member states in the WHO South East Asia Region. It is expected that with the landslide victory of the National League for Democracy in the recent general election, new government is very likely to reform the health system to meet the needs of the country and people expectation, there will be positive change in the health expenditure and in the implementation for the Universal Health Coverage, and hence, the funding and implementation of diabetes care model will be made feasible through these changes.

\section{Community mobilization}

Prior to the implementation at each primary healthcare level, advocacy on the healthy lifestyles, health and socioeconomic burden of diabetes and other major noncommunicable diseases, prevention and control of noncommunicable diseases including diabetes, and services 
Table 2. Functions of diabetes care according to the healthcare level

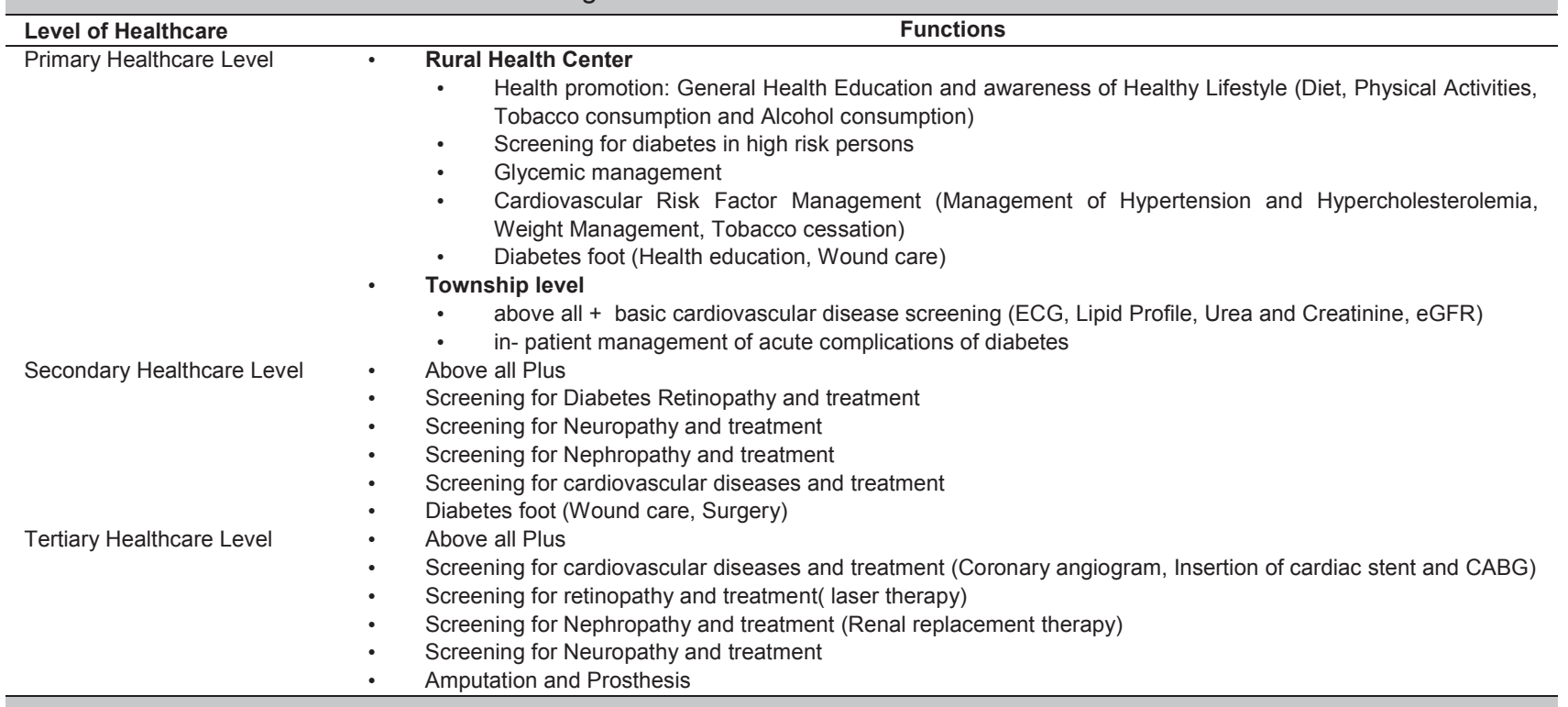

available at each health center for standard of diabetes care, will be conducted to the local administrative authorities, community leaders and local nongovernmental organizations. The purpose of the advocacy

Table 3. Essential drugs and equipment of diabetes care according to the healthcare level

\begin{tabular}{|c|c|}
\hline $\begin{array}{l}\text { Level of } \\
\text { Healthcare }\end{array}$ & Essential drugs and equipment \\
\hline Primary & Drugs \\
\hline Healthcare & - Metformin \\
\hline \multirow[t]{23}{*}{ Level } & - $\quad$ Gliclazide \\
\hline & - Insulin \\
\hline & - Hydrochlothiazide \\
\hline & - Amlodipine \\
\hline & - Atenolol \\
\hline & - $\quad$ Atorvastatin \\
\hline & - Isosorbide dinitrate \\
\hline & - Glyceryl trinitrate \\
\hline & - $\quad$ Furosemide \\
\hline & - $\quad$ Spironolactone \\
\hline & - $\quad$ Aspirin \\
\hline & - Equipment \\
\hline & - Thermometer \\
\hline & - $\quad$ Stethoscope \\
\hline & - $\quad$ Blood pressure measurement device* \\
\hline & - $\quad$ Measurement tape \\
\hline & - Weighing machine \\
\hline & - $\quad$ Glucometer \\
\hline & - $\quad$ Blood glucose test strips \\
\hline & - Lancets and needles \\
\hline & - Urine protein test strips \\
\hline & - Urine ketones test strips \\
\hline & - $\quad$ Electrocardiograph \\
\hline Secondary & - $\quad$ Pulse oximeter \\
\hline Healthcare & Lipid profile assay \\
\hline \multirow[t]{5}{*}{ Level } & - $\quad$ Serum creatinine assay \\
\hline & - $\quad$ Troponin test strips \\
\hline & - $\quad$ Urine microalbuminuria test strips \\
\hline & - $\quad$ Tuning fork \\
\hline & - Defibrillator \\
\hline Tertiary & Advanced technologies for specialist \\
\hline Healthcare & - $\quad$ Laser therapy \\
\hline \multirow[t]{5}{*}{ Level } & - Haemodialysis \\
\hline & - $\quad$ Continuous Ambulatory Peritoneal dialysis \\
\hline & - $\quad$ Neck Lines \\
\hline & - $\quad$ Angiogram \\
\hline & - Doppler Ultrasound \\
\hline
\end{tabular}

is to mobilize the community to participate in the prevention and control of diabetes and other noncommunicable diseases. Previous experience with the pilot project on the PEN implementation, it was proved that the community mobilization to motivate the public to participate in the activities of the project is feasible. Health seeking behavior of the public can be changed to facilitate the screening for the high risk persons for diabetes and cardiovascular diseases and so is the feasibility of the community participation in the adoption of healthy lifestyles, in particular, increased mass physical activity and raising of local fund for the sustenance of the activities through local donations by generous and philanthropic persons in the localities.

\section{Development of IEC materials}

It is also necessary to develop the advocacy tool kits for stakeholders, and also information, education and communication (IEC) materials for health education diabetes care for the public. With the widespread

Table 4. Required health personnel for diabetes care according to the healthcare level

\begin{tabular}{lll}
\hline $\begin{array}{l}\text { Level of } \\
\text { Healthcare }\end{array}$ & & \multicolumn{1}{c}{ Functions } \\
\hline Primary & - & Midwife \\
Healthcare & - & Health Assistant \\
Level & - & Medical Officer \\
Secondary & - & Above all PLUS \\
Healthcare & - & Specialist (Medicine, Surgery, OG and \\
Level & & Pediatrician) \\
& - & Diabetes educator \\
Tertiary & - & Above all PLUS \\
Healthcare & - & Endocrinologists \\
Level & - & Orthopaedic and Vascular surgeons \\
& - & Diabetes Specialist Nurse \\
& - Nutritionist \\
& - & Podiatrist \\
& - & Physical Activity instructor \\
& - Occupational therapist \\
& &
\end{tabular}


dissemination of information on the diabetes care model and availability of these services at the local RHC and SubRHC and township hospitals public can be informed as well as motivated to participate in the project activities and utilize the health services in their local health centers.

\section{Development of guidelines}

It is also important to develop guidelines for the delivery of standard diabetes care at different healthcare levels. Guidelines for the BHS and medical officers will be developed differently and these guidelines can be used as manuals for them in daily implementation of the activities of the diabetes care model. A registry with minimal essential data for evaluation, monitoring and reporting is also necessary to be developed.

\section{Capacity building of Health Work Force}

It is mandatory to build the capacity of basic health staff from the primary healthcare levels and medical doctors from the private clinic on the standard of diabetes care and proper referral according to the guidelines developed for the diabetes care model. Capacity building workshops for township medical officers will be conducted as training of trainers for each township. TMO will conduct multiplier workshops for the BHS and medical officers from both public and private sectors in their townships. Junior physicians from the district and State and Regional hospitals will also need to strengthen their capacity on delivery of standard of diabetes care.

\section{Monitoring and evaluation}

Regular monitoring and evaluation of the progress in each township will be done and findings will also be utilized for further advocacy to the stakeholders to strengthen their sustained collaboration and cooperation in the delivery of diabetes care. The NCD Unit of the Department of Health will collect regular reports and returns from States and Region Health Offices to monitor the progress achieved in the whole country.

\section{CONCLUSION}

Since diabetes is one of the major non-communicable diseases with shared common risk factors, implementation of the MMDCM should be carried out in aligned with the national action plan for the prevention and control of other non-communicable diseases, which is already endorsed by the National Health Committee Chaired by the Vice President of Republic of the Union of Myanmar.

The Myanmar Diabetes Care Model aims to reduce the inequity in the delivery of diabetes care in urban and rural population. Ultimately it is aimed to lessen the health and socioeconomic burden of diabetes in Myanmar. Achievement of its goal will certainly contribute to the poverty alleviation and national development of the country. Collaboration and cooperation of all stakeholders involved in the diabetes care is the key for success. Although Ministry of Health will be the focal for the implementation, collaboration of other sectors and partners like academia, civic societies and community is obviously necessary for the successful implementation.

Successful implementation of the care model will certainly facilitate Myanmar to achieve diabetes related targets and indicators set in the Global Action Plan for the Prevention and Control of non-communicable diseases (2013-2020). It is also expected that reduction in the burden caused by diabetes and other major NCD will eventually contribute to the national development of Myanmar.

\section{References}

1. Vinicor F, Rufo K, Murphy D. Diabetes and public health in the United States. In: International Textbook of Diabetes Mellitus, 3rd ed. Zimmer P. Chichester, West Sussex, UK: John Willey \& Sons, 2004, pp. 1785-1792.

2. International Diabetes Federation. IDF Diabetes Atlas, sixth edition, 2013. www.idf.org/diabetesatlas, pp. 1-160.

3. Soe P, Latt TS, Aung PP Myint TK. Glucose intolerance and associated factors in four townships of Yangon Division: STEP Survey of Yangon Division. DMR Research Congress, 2004.

4. Non-communicable disease risk factors survey Myanmar 2009: http://www.who.int/chp/steps/2009-STEPS -Survey-Myanmar. pdf . Accessed on September 20, 2015.

5. Latt TS, Ko K, Zaw KK. National Survey on the prevalence of diabetes and risk factors for Non-Communicable diseases, 2014. (personal communications)

6. World Health Organization 2013. Global Action Plan for the prevention and control of NCDs 2013-2020. http://apps.who.int/iris/ bitstream/10665/94384/1/9789241506236_eng.pdf?ua=1. pp. 1-103.

7. Political declaration of the high-level meeting of the General Assembly on the prevention and control of non-communicable diseases, 66th Session of the General Assembly, agenda item 117. A/66/L. 1, 2011.

8. World Health Organization. Global status report on noncommunicable diseases. Tech. Rep., World Health Organization, Geneva, Switzerland, 2010.

9. World Health Organization. The global strategy for prevention and control of non-communicable diseases. Tech. Rep. Resolution WHA 53. 14. World Health Organization, Geneva, Switzerland, 2000.

10. World Health Organization. 2008-2013 action plan for the global strategy for prevention and control of non-communicable diseases. Tech. Rep. Resolution WHA61.14. World Health Organization, Geneva, Switzerland.

11. Abegunde DO, Shengelia B, Luyten A, et al. Can non-physician health-care workers assess and manage cardiovascular risk in primary care? Bull World Health Organ. 2007;85(6):432-440.

12. World Health Organization. Prevention of cardiovascular disease: Guidelines for assessment and management of cardiovascular risk. World Health Organization, Geneva, Switzerland, 2007.

13. World Health Organization. Scaling up action against noncommunicable diseases. World Health Organization, Geneva, Switzerland, 2011.

14. World Health Organization. Package of Essential Non-communicable Disease Interventions for primary healthcare in low-resource settings, World Health Organization, Geneva, Switzerland, 2010.

15. World Health Organization. The World Health report, health systems financing: The path to Universal Coverage. World Health Organization, Geneva, Switzerland, 2010.

16. World Health Organization. Report of the Commission on Macroeconomics and Health: investing in Health for Economic Development (WHO-CMH). Tech. Rep. World Health Organization, Geneva, Switzerland, 2001.

17. Sein TT, Myint P, Tin N, Win H, Aye S, Sein T. The Republic of the Union of Myanmar Health system review: Health system in transaction. Asia Pacific Observatory on Health Systems and Policies. 2014;4(3).

18. Mendis S, Bashir IA, Dissanayake L, et al. Gaps in capacity in primary care in low-resource settings for implementation of Essential Noncommunicable Disease Interventions, International Journal of Hypertension. 2012;2012. 
19. Aye TT, Aung MW, Oo ES. Diabetes mellitus in Myanmar: Sociocultural challenges and strength. J Soc Health Diabetes. 2014;2(1):9-13. http://dx.doi.org/10.4103/2321-0656.120255.

20. Hass L, Maryniuk M, Berk I, et al. National Standard for diabetes selfmanagement education and support. Diabetes Care. 2013;36(Suppl1):S100-8.http://dx.doi.org/10.2337/dc13-S100.

21. American Diabetes Association. Standards of medical care in diabetes-2015: Summary of revisions. 2015;38(Suppl 1):S4. http://dx.doi.org/10.2337/dc15-S003.

22. Wagner EH, Austin BT, Von Korff M. Improving outcomes in chronic illness. Manag Care Q. 1996;4(2):12-25.

23. Bodenheimer $\mathrm{T}$, Wagner $\mathrm{EH}, \mathrm{Grumbach} \mathrm{K}$. Improving primary care for patients with chronic illness: The chronic care model, part 2. JAMA. 2002;288(15):1775-79.

24. Wagner EH. The role of patient care teams in chronic disease management. BMJ. 2000;320:569-572. http://dx.doi.org/10.1136/ bmj.320/7234.569.

25. Wagner EH. Meeting the needs of chronically ill people. BMJ. 2001;323:945-946. http://dx.doi.org/10.1136/bmj.323.7319.945.
26. Wagner EH, Grothaus LC, Sandhu N, Galvin MS, McGregor M, Artz $\mathrm{K}$, Coleman EA. Chronic care clinics for diabetes in primary care: A system-wide randomized trial. Diabetes Care. 2001;24(4):695-700. http://dx.doi.org/10.2337/diacare.24.4.695.

27. Stellefson M, Dipnarine K, Stopka C. The chronic care model and diabetes management in US primary care settings: A systematic review. Prev Chronic Dis. 2013;10:120180. http://dx.doi.org/ 10.5888/pcd10.120180.

28. Wagner EH. High quality care for people with chronic disease (Editorial). BMJ. 2005;330:609-610. http://dx.doi.org/10.1136/bmj. 330.7492.609.

29. World Health Organization. Prevention and control of noncommunicable diseases: Guidelines for primary healthcare in lowresource settings. Geneva, WHO. 2012, pp. 9-11.

30. Ministry of Health, Myanmar: Pilot project for implementation of Package of Essential Noncommunicable (PEN) diseases interventions in Hlegu and Hmawbi townships, 2012-2013. (personal communications)

Articles and any other material published in the JAFES represent the work of the author(s) and should not be construed to reflect the opinions of the Editors or the Publisher. Authors are required to accomplish, sign and submit scanned copies of the JAFES Declaration: that the article represents original material, that is not being considered for publication or has not been published or accepted for publication elsewhere. Consent forms, as appropriate, have been secured for the publication of information about patients; otherwise, authors declared that all means have been exhausted for securing such consent. The authors have signed disclosures that there are no financial or other relationships that might lead to a conflict of interest. All authors are required to submit Authorship Certifications that the manuscript has been read and approved by all authors, and that the requirements for authorship have been met by each author.

\section{Appendices}

\section{Appendix 1: Voluntary Global Targets ${ }^{6}$}

1. A $25 \%$ relative reduction in risk of premature mortality from cardiovascular diseases, cancer, diabetes, or chronic respiratory diseases.

2. At least $10 \%$ relative reduction in the harmful use of alcohol, as appropriate, within the national context.

3. A $10 \%$ relative reduction in prevalence of insufficient physical activity.

4. A $30 \%$ relative reduction in mean population intake of salt/sodium.

5. A $30 \%$ relative reduction in prevalence of current tobacco use in persons aged $15+$ years.

6. A $25 \%$ relative reduction in the prevalence of raised blood pressure or contain the prevalence of raised blood pressure, according to national circumstances.

7. Halt the rise in diabetes and obesity.

8. At least $50 \%$ of eligible people receive drug therapy and counselling (including glycaemic control) to prevent heart attacks and strokes.

9. An $80 \%$ availability of the affordable basic technologies and essential medicines, including generics, required to treat major noncommunicable diseases in both public and private facilities. 
Appendix 2: Protocol for the management of diabetes and cardiovascular risk factors at primary care ${ }^{18}$ When could this Protocol be used?

- The protocol is for assessment and management of cardiovascular risk using hypertension, diabetes mellitus (DM) and tobacco use as entry points

- It could be used for routine management of hypertension and DM and for screening, targeting the following categories of people:

- $\quad$ age $>40$ years

- $\quad$ smokers

- waist circumference ( $\geq 90 \mathrm{~cm}$ in women $\geq 100 \mathrm{~cm}$ in men)

- known hypertension

- known DM

- history of premature CVD in first degree relatives

- history of DM or kidney disease in first degree relatives

\section{Follow instructions given in Action 1 to Action 4, step by step}

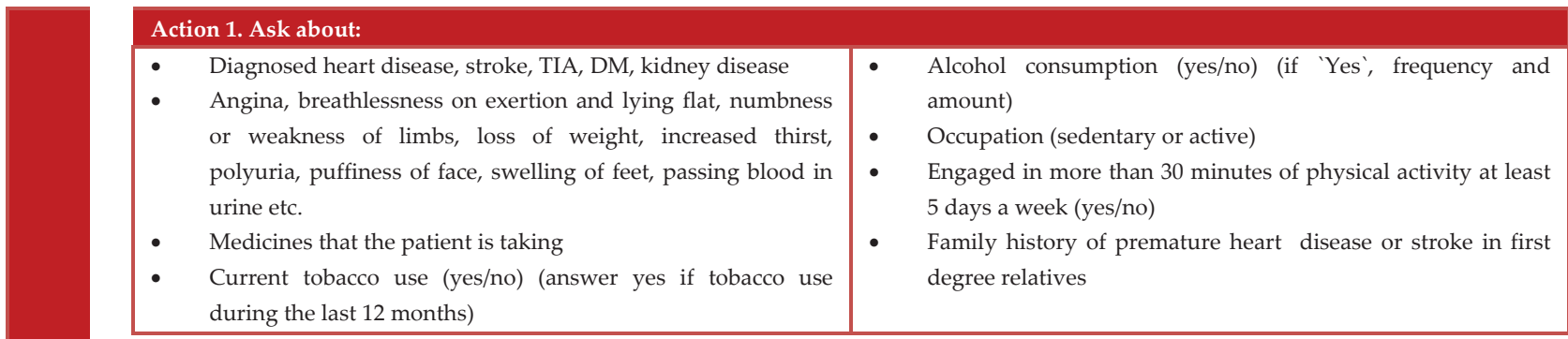

Action 2. Assess (physical exam and blood and urine tests):

- Waist circumference

- Measure blood pressure, look for pitting oedema

- Palpate apex beat for heaving and displacement

- Auscultate heart (rhythm and murmurs)

- Auscultate lungs (bilateral basal crepitations)

- Examine abdomen (tender liver)

- In DM patients examine feet; sensations, pulses, and ulcers
- $\quad$ Urine ketones (in newly diagnosed DM) and protein

- Total cholesterol

- Fasting or random blood sugar (diabetes= fasting blood sugar $\geq 7 \mathrm{mmol} / \mathrm{l}(126 \mathrm{mg} / \mathrm{dl})$ ) or random blood sugar $\geq 11 . \mathrm{i} \mathrm{mmol} / \mathrm{l}$ $(200 \mathrm{mg} / \mathrm{dl}))$

(Point of care devices can be used for testing blood sugar if laboratory facilities are not available)

\section{Action 3. Estimate cardiovascular risk (in those not referred):}

- Use the WHO/ISH risk charts relevant to the WHO sub region (Appendix 4)

- $\quad$ Use age, gender, smoking status, systolic blood pressure, DM (and plasma cholesterol if available)

- If age 50-59 years select age group box 50, if 60-69 years select age group box 60 etc., for people age $<40$ years select age group box 40

- If cholesterol assay cannot be done use the mean cholesterol level of the population or a value of $5.2 \mathrm{mmol} / 1$ to calculate the cardiovascular risk)

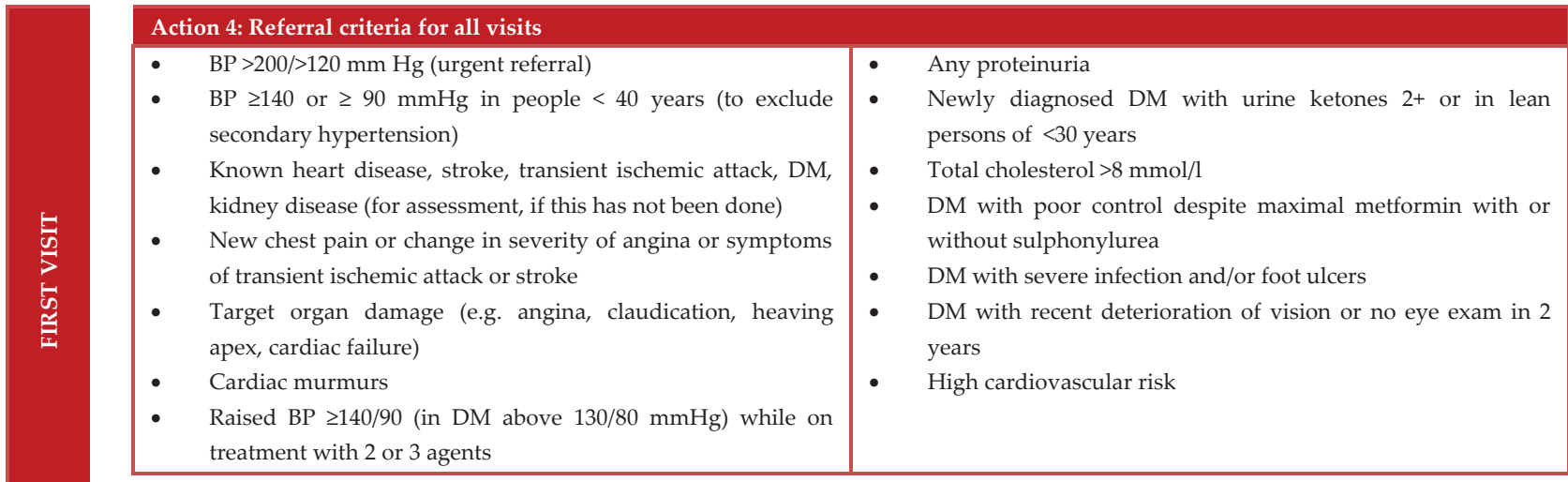




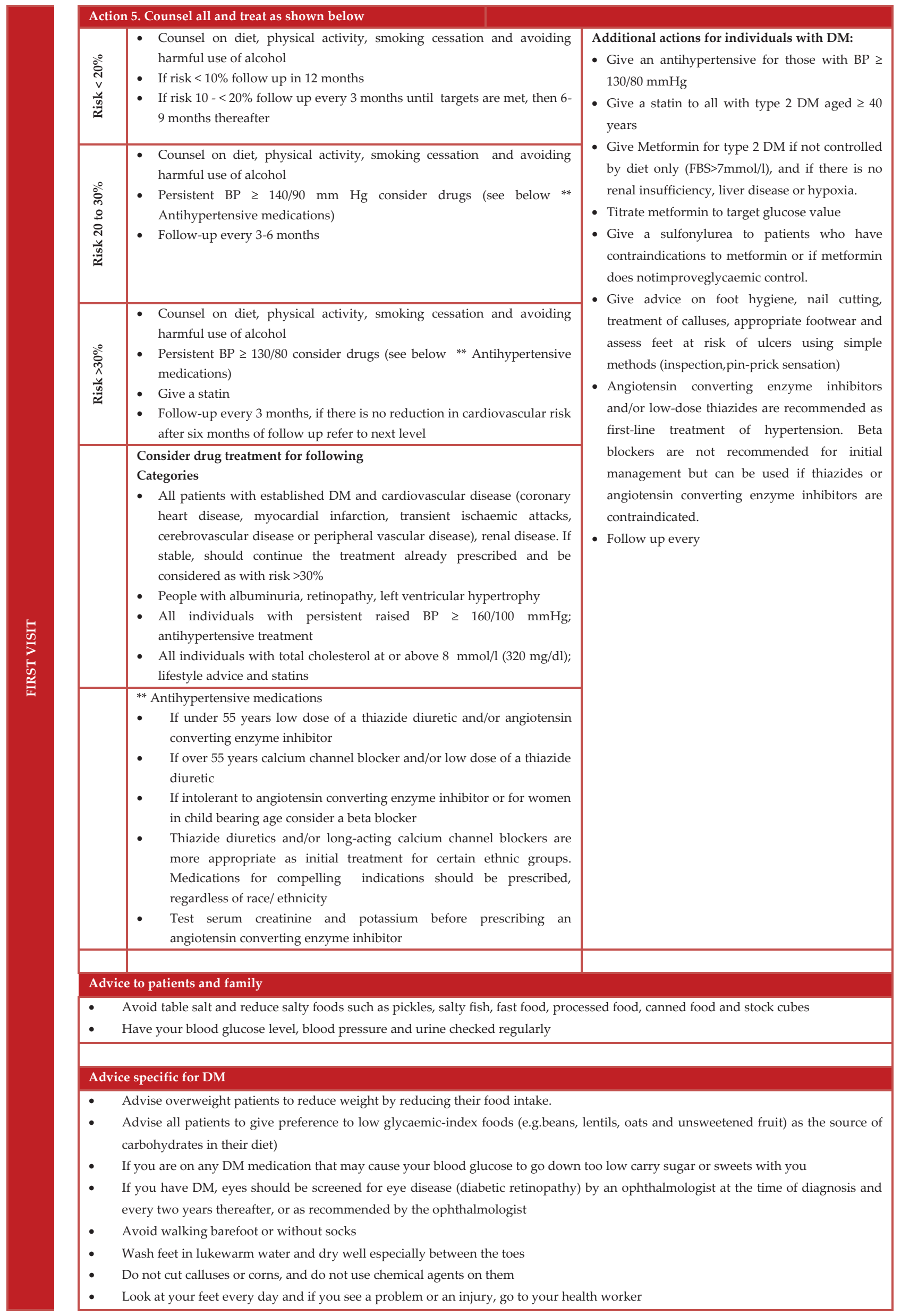


- Action 3 Estimate cardiovascular risk

- Action 4 Refer if necessary

- Action 5 Counsel all and treat as shown in protocol

Appendix 3: Protocol for Health Education and Counseling on Healthy Behaviours ${ }^{18}$

\section{Educate your patient to}

- $\quad$ Take regular physical activity

- Eat a "heart healthy" diet

- Stop tobacco and avoid harmful use of alcohol

- Attend regular medical follow-up

\section{Take regular physical activity}

- $\quad$ Progressively increase physical activity to moderate levels (such as brisk walking); at least 150 minutes per week

- Control body weight and avoid overweight by reducing high calorie food and taking adequate physical activity

\section{Eat a heart healthy diet}

Salt (sodium chloride)

- Restrict to less than 5 grams (1 teaspoon) per day

- $\quad$ Reduce salt when cooking, limit processed and fast foods Fruits and vegetables

- 5 servings (400-500 grams) of fruits and vegetable per day

- 1 serving is equivalent to 1 orange, apple, mango, banana or 3 tablespoons of cooked vegetables

Fatty food

- Limit fatty meat, dairy fat and cooking oil (less than two tablespoons per day)

- Replace palm and coconut oil with olive, soya, corn, rapeseed or safflower oil

- $\quad$ Replace other meat with chicken (without skin)

\section{Stop Tobacco and avoid harmful use of Alcohol:}

- $\quad$ Encourage all non-smokers not to start smoking

- Strongly advise all smokers to stop smoking and support them in their efforts

- Individuals who use other forms of tobacco should be advised to quit

- Alcohol abstinence should be reinforced.

- People should not be advised to start taking alcohol for health reasons

- Advise patients not to use alcohol when additional risks are present, such as:

- driving or operating machinery

- pregnant or breast feeding

- $\quad$ taking medications that interact with alcohol

- having medical conditions made worse by alcohol

- $\quad$ having difficulties in controlling drinking

\section{Adherence to treatment}

- If the patient is prescribed a medicine/s:

- teach the patient how to take it at home:

- $\quad$ explain the difference between medicines for long- term control (e.g. blood pressure) and medicines for quick relief (e.g. for wheezing)

- tell the patient the reason for prescribing the medicine/s

- Show the patient the appropriate dose

- Explain how many times a day to take themedicine

- Label and package the tablets

- Check the patient's understanding before the patient leaves the health centre

- Explain the importance of:

- $\quad$ keeping an adequate supply of the medications

- the need to take the medicines regularly

- $\quad$ as advised even if there are no symptoms
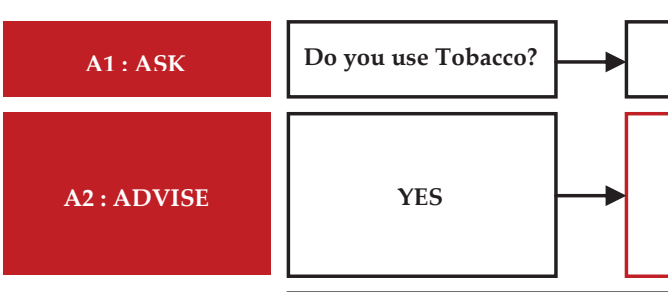

A3 : ASSESS

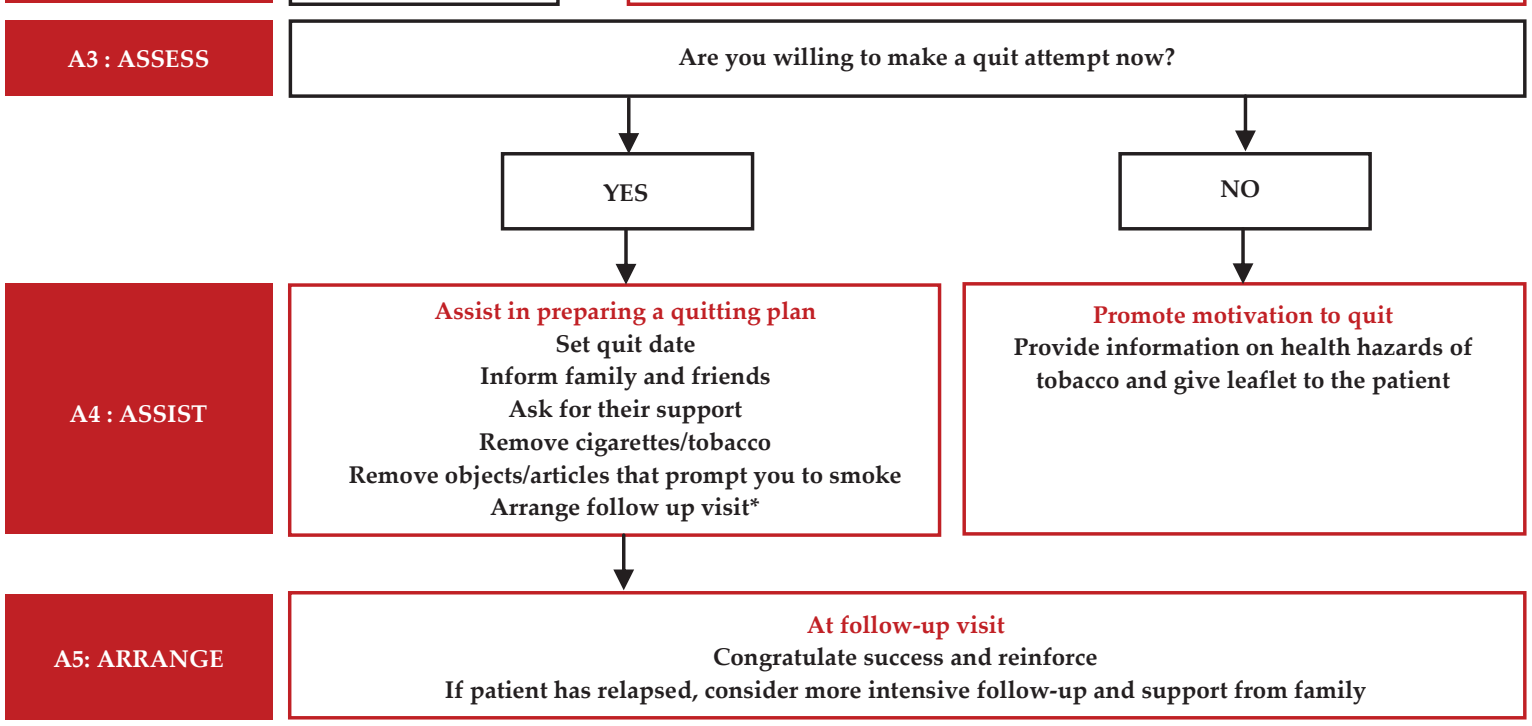

A5: ARRANGE

Advise to quit in a clear, strong and personalized manner "Tobacco use increases the risk of developing a heart attack, stroke, lung cancer and respiratory diseases. Quitting tobacco use is the one most important hing you can do to protect your heart and health, you have to quit now."
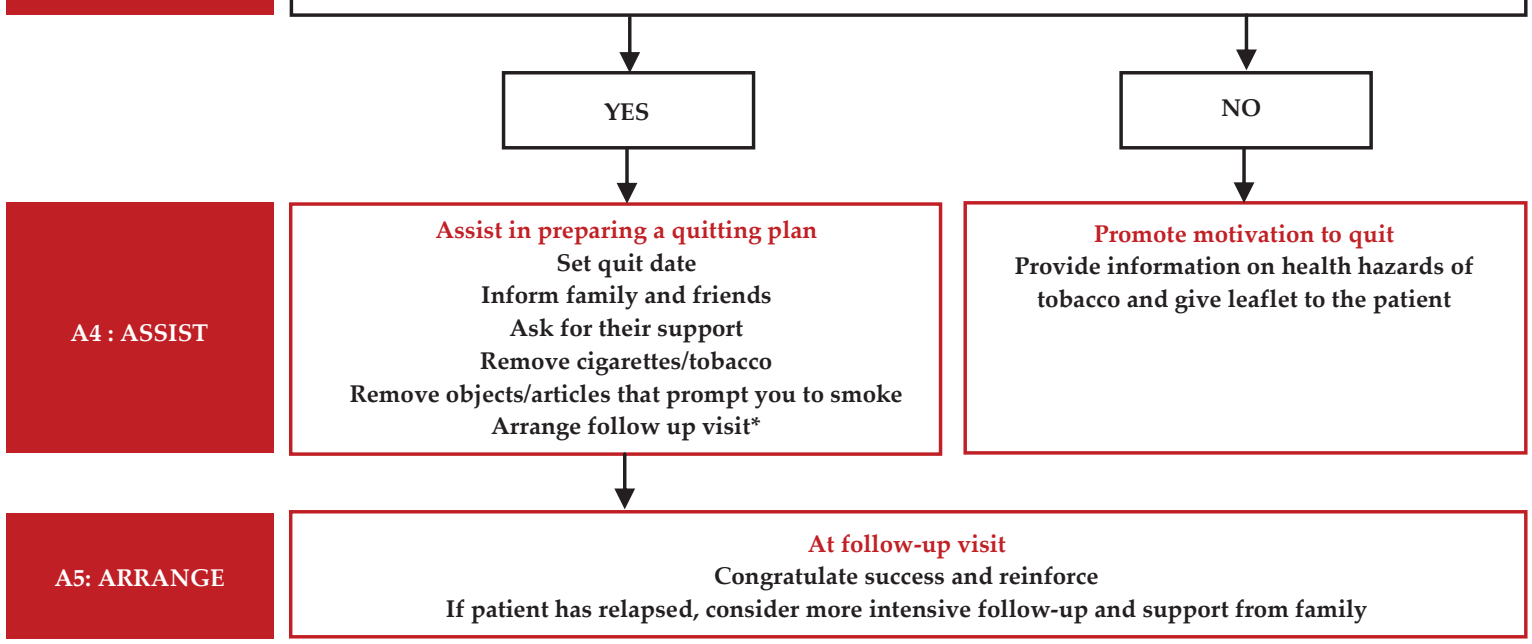

Reinforce message that tobacco increases risk of heart disease

Source: World Health Organization Implementation tools, Package of Essential Noncommunicable (PEN) disease interventions for primary healthcare in low-resource settings 
Appendix 4-a: WHO/ISH risk prediction chart. 10-year risk of a fatal or non-fatal cardiovascular event by gender, age, systolic blood pressure, total blood cholesterol, smoking status and presence of diabetes mellitus ${ }^{18}$

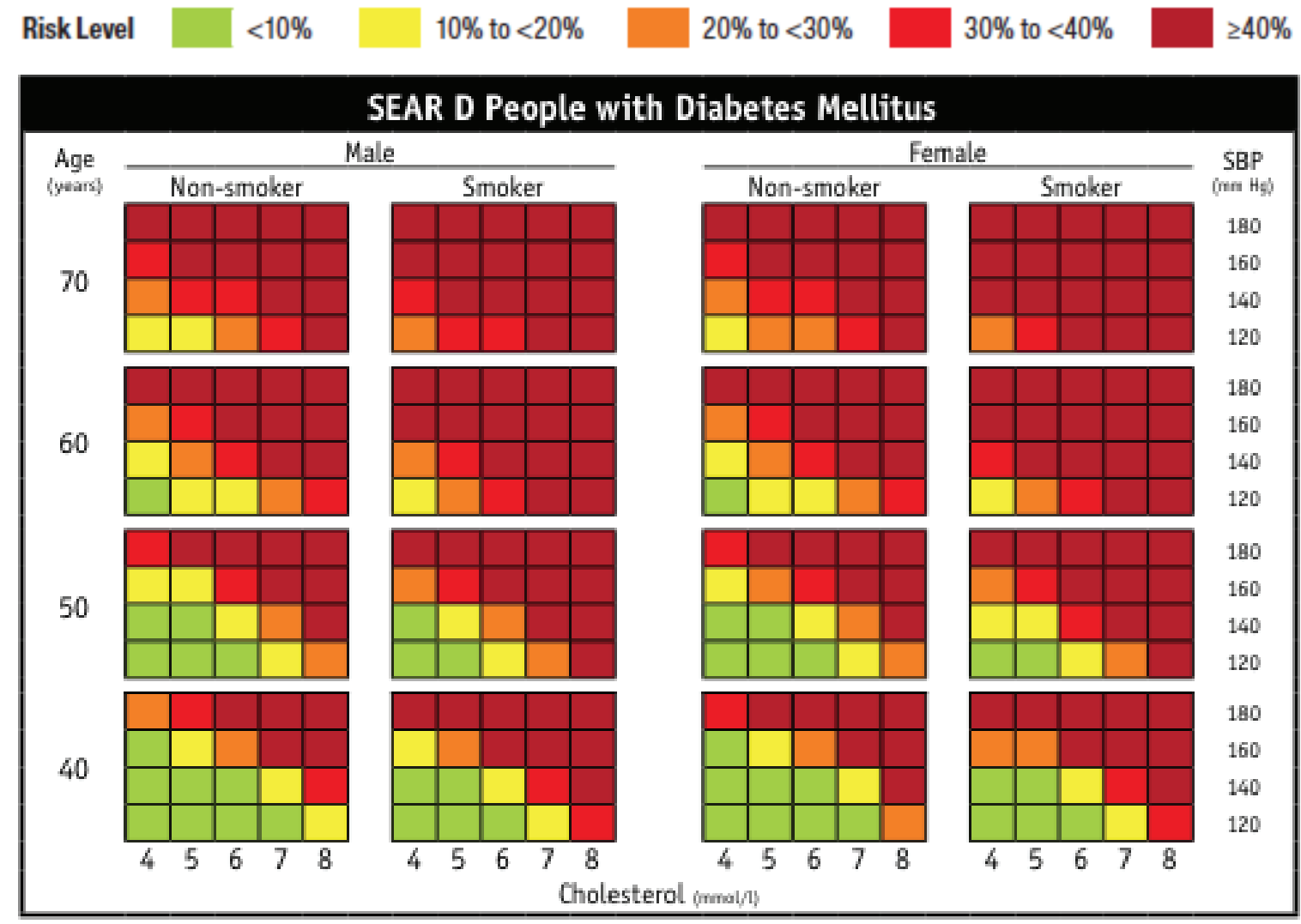

Appendix 4-b: WHO/ISH risk prediction chart. 10-year risk of a fatal or non-fatal cardiovascular event by gender, age, systolic blood pressure, total blood cholesterol, smoking status and absence of diabetes mellitus 18

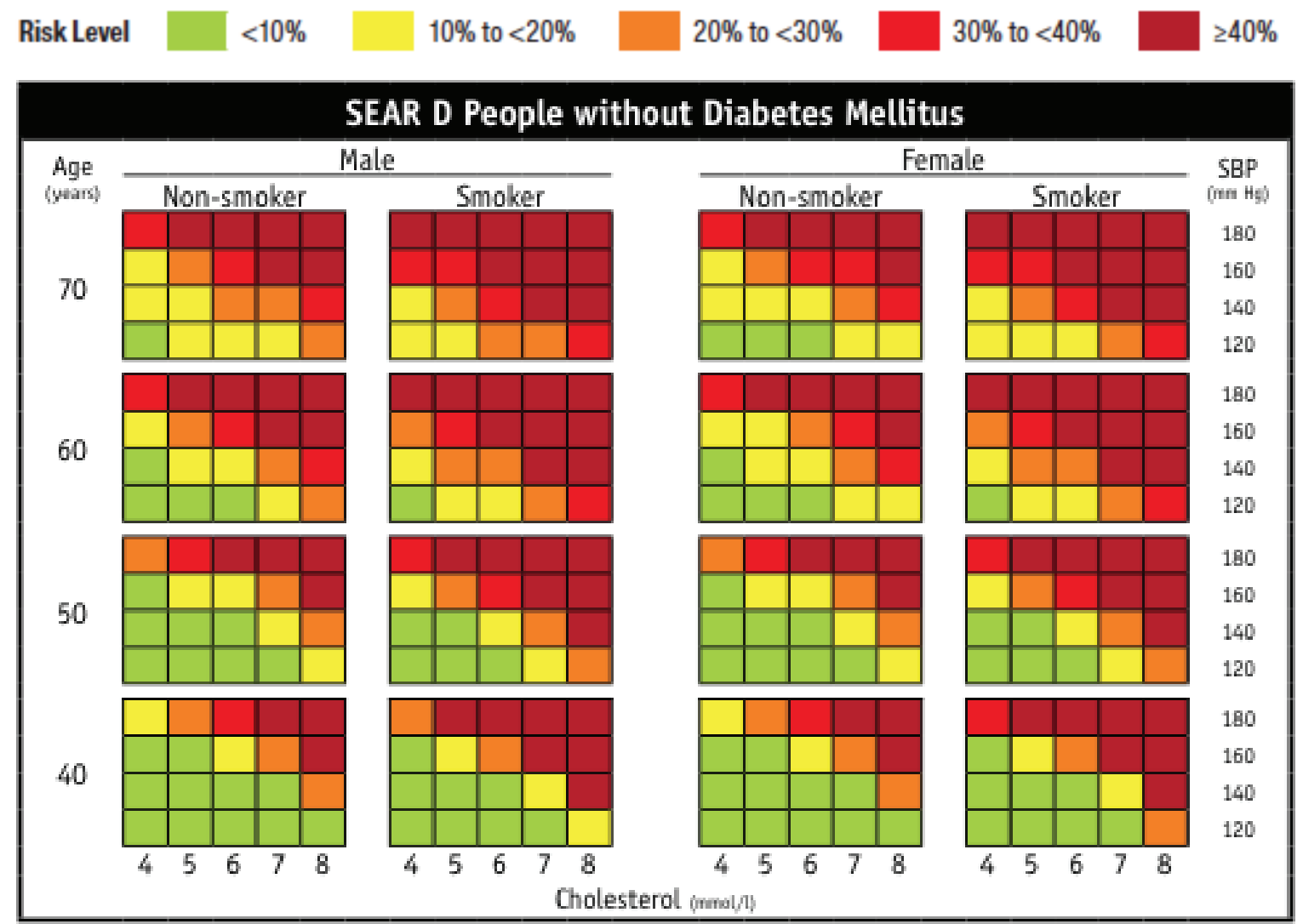

